
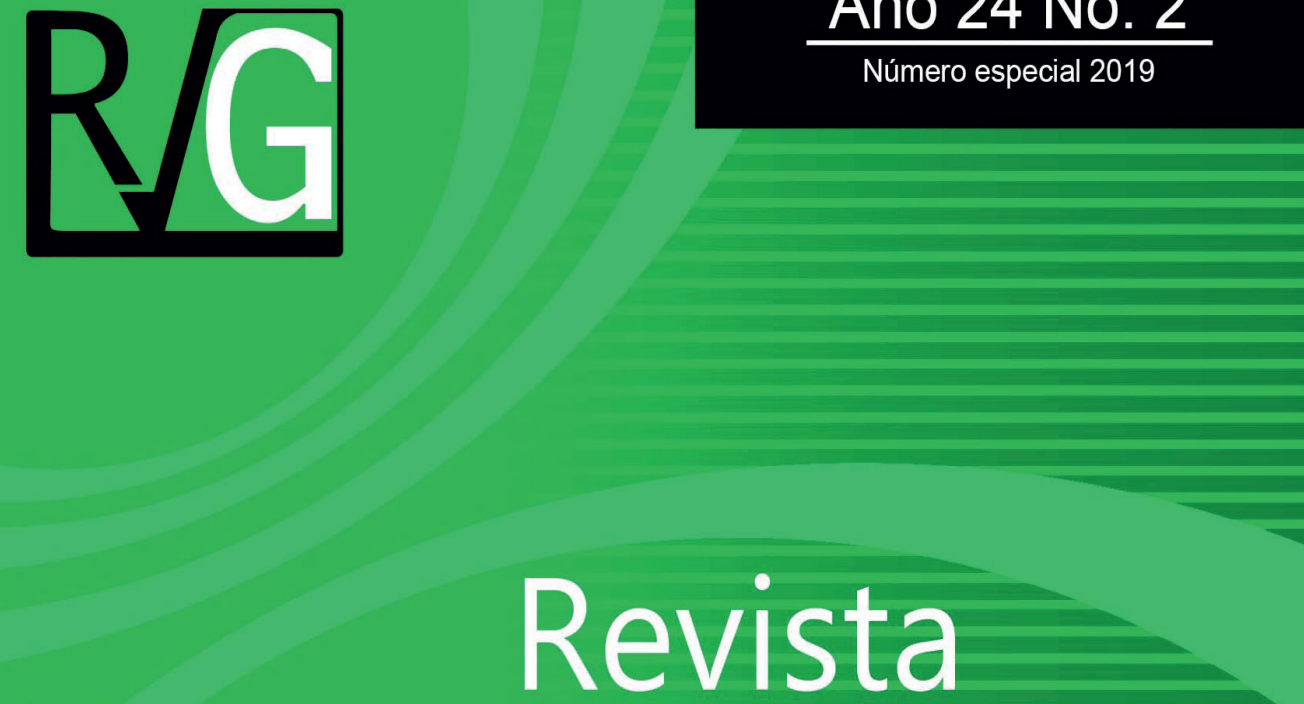

Venezolana de

$\frac{1}{6}$

$\frac{\mathbb{O}}{\frac{1}{0}}$
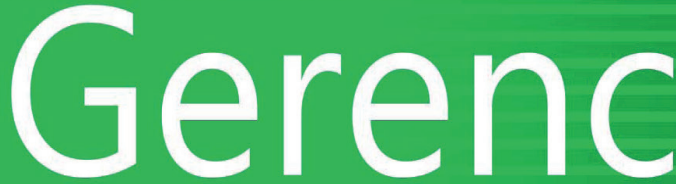

0

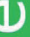

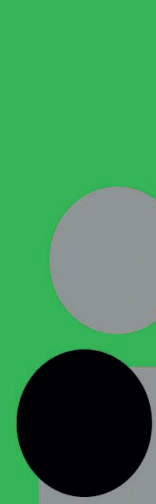

ro

u

$>$

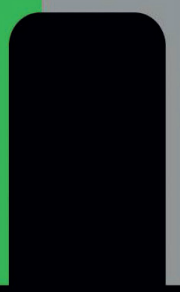




\title{
Tecnología digital y afectaciones a la cultura de aprendizaje de sujeto social ${ }^{1}$
}

\author{
Rodriguez-Cadena, Rodolfo ${ }^{2}$
}

\section{Resumen}

Ante los cambios generados por la revolución tecnológica en los años 80 s el objetivo del estudio busca identificar las afectaciones a la cultura de aprendizaje del sujeto social de nuevas generaciones. A través de una investigación cualitativa fundamentada en las teorías de complejidad y pensamiento complejo, mediante una revisión bibliométrica integrativa en las bases de datos Redalyc, Scielo, Dialnet, Wos y Scopus. Dentro de los hallazgos se destaca la incidencia del uso de la tecnología digital en los desequilibrios patológicos del sujeto social como la adición, ansiedad, depresión, perdida de realidad y apatía al aula de clase. Finalmente, se destaca el deterioro de autocontrol del sujeto en contenidos publicados en web, así como una creciente influencia negativa del Internet en los cambios socio-culturales de las nuevas generaciones.

Palabras clave: tecnología digital; cultura; sujeto social, complejidad.

El presente artículo es un avance de investigación de la tesis de doctorado en pensamiento Complejo de Multiversidad Mundo Real Edgar Morin - Hermosillo, Sonora, México.

2 Investigador Universidad Sergio Arboleda y Director del grupo de investigación entorno económico y competitividad GEECO - categoría A1 Colciencias convocatoria 833 de 2018, Universidad Sergio Arboleda Colombia. - Ingeniero de Sistemas, Especialista en Redes y Servicios Telemáticos Universidad Manuela Beltrán Bogotá, Especialista en Docencia e Investigación de Universidad Sergio Arboleda. ORDCID: 0000-0001-7729-4906

Mis agradecimientos a Universidad Sergio Arboleda por el apoyo financiero a la investigación. 


\title{
Digital technology and effects on the learning culture of the social subject
}

\begin{abstract}
Given the changes generated by the technological revolution in the 80 s, the objective of the study seeks to identify the effects on the culture of learning of the social subject of new generations. Through qualitative research based on theories of complexity and complex thinking, through an integrative bibliometric review of the Redalyc, Scielo, Dialnet, Wos and Scopus databases. Among the findings, the incidence of the use of digital technology in the pathological imbalances of the social subject such as addition, anxiety, depression, loss of reality and apathy to the classroom is highlighted. Finally, the deterioration of self-control of the subject in content published on the web, as well as a growing negative influence of the Internet in the socio-cultural changes of the new generations is highlighted.
\end{abstract}

Keywords: digital technology; culture; social subject; complexity

\section{Introducción}

La ciencia en los últimos 40 años ha contribuido con la creación de la revolución tecnológica digital incidiendo significativamente en el cambio de comportamiento del homo sapiens en forma positiva así como negativa; sin embargo, estos cambios fueron saltos paulatinos a finales de los 70 y la actualidad. Inicialmente, fue creación del computador personal, el desarrollo de World Wide Web (red mundial de informática), las redes sociales, los teléfonos inteligentes y las aplicaciones en pleno siglo XXI. Estos fueron avances de la ciencia y la tecnología, causantes de las transformaciones del sujeto, la sociedad y la cultura de aprendizaje de las nuevas generaciones.

En este contexto, Maturana y Varela (1994) manifiestan que, cuando los seres vivos se les intervienen el nicho ecológico de su entorno natural sufren afectaciones con resultados impredecibles frente a su disposición relacional con la especie. En este sentido, la disrupción de la tecnología en el sujeto social involucra conocer y comprender la complejidad humana. Puesto que, el estudio del sujeto conlleva abordarlo desde interdisciplinar, multidisciplinar y transdisciplinar Morin (1977). Así mismo, Morin (1984) sostiene que hombre es una unidad de múltiples dimensiones que se integra desde lo biológico de los sentidos, psicológico de las emociones, socio-cultural del comportamiento y de los procesos emergencia de la conciencia.

Considerando que, somos parte de un nuevo florecimiento de la humanidad a quien Bell (1973) llama la sociedad de la información la cual es transitada por dos generaciones de individuos llamados migrantes y nativos digitales (Presky, 2010). No obstante, los ciudadanos nacidos en la era digital se han visto 
afectados por dispositivos tecnológicos con acceso al internet han generado problemas serios de aprendizaje a causa de su mal uso. Aunque es obvio manifestar que, la tecnología digital ha facilitado las interrelaciones entre seres humanos de diferentes culturas produciendo un salto evolutivo de la especie. No obstante, su mal uso y abuso de estas tecnológicas son también el inicio de afectaciones al ser humano causantes desorden y caos en la cultura de aprendizaje de la sociedad como lo expresa (Castells, 2012).

Cabe señalar que, la ciencia mediante el desarrollo de la tecnología ha creado un mundo interconectado como resultado este planeta se ha convertido en una aldea global que intercambia información constantemente (McLuhan, 1995). Es necesario entender que, la sociedad es un sistema complejo integrado por sujetos que interactúan y se relacionan. Por lo tanto, emerge un pensamiento colectivo que de acuerdo con (Cuzin, 2007) genera nuevos patrones de comportamiento y cambios de conducta en el sujeto y la sociedad. Sin embargo, es oportuno señalar que existen sociedades cerradas y aisladas, como grupos de indígenas en Colombia que habitan zonas de la alta Guajira o el Amazonas, en donde, no hay contagio de ideas y ni comportamientos que incidan en cambios de costumbres del sujeto (Parejo et al, 2018:09)

En este orden de ideas, se justifica esta investigación en la medida que existía la necesidad de comprender y reflexionar sobre un problema complejo, creciente y que involucra individuos que forman parte de una sociedad cambiante. Así mismo, es describir como los cambios del paradigma tecnológico está ocasionando una ruptura en la sociedad que inciden en hábitos y costumbres de las nuevas generaciones. Teniendo en cuenta que, la sociedad es un sistema complejo de relaciones e interrelaciones entre sujetos que originan patrones de comportamiento e inciden en la creación de nuevos modelos mentales del sujeto (Senge, 1995:30).

Debemos comprender que, hay carencia de estudios bibliométrico sobre las redes de bases de datos científicas. Por tanto, es ineludible hacer un análisis de los datos y posteriormente realizar una síntesis de los resultados de las afectaciones generadas por la tecnología y su incidencia en el sujeto social y el desequilibrio causado en su entorno natural. No obstante, debe quedar bastante claro que el presente estudio buscó responder la pregunta de investigación de ¿cómo la tecnologías digital y el uso de internet afectan la cultura de aprendizaje de nuevas generaciones?,

En las anteriores palabras se debe advertir de la necesidad de hacer un análisis y síntesis de los datos (Maldonado, 2009) desde la una postura de la complejidad advierte que la verdadera genialidad radica en saber interpretar los datos en donde emergen estructuras que forman patrones de comportamiento de sistemas vivos; de hecho el sistema de investigadores agrupados espontáneamente por relaciones de intereses comunes preocupados por las afectaciones del mal uso de la tecnología. Por lo tanto, se pretendió a través de una búsqueda bibliométrica determinar las consecuencias a través de publicaciones científicas que abordan temas relacionados con afectaciones al ser humano desencadenado como resultado el ruido o desorden de la información que es asimilada por la estructura cognitiva del sujeto (Piaget, 1991). 
Dentro de este entorno, se destacan estudios como los de (MuñozDueñas et al, 2017) quienes consideran que el uso de internet interfiere en las actividades cotidianas del ser humano, al generar malas habilidades sociales, pérdida de calidad del sueño, los cuales determinan patrones de conductas patológicas impulsivas, agresividad, síntomas somáticos, ansiedad, insomnio, aislamiento social y depresión.

En un sentido similar, las investigaciones realizadas por (Pérez et al, 2018:19-32) manifiestan que, las nuevas generaciones están siendo afectados por el uso y abuso de dispositivos electrónicos, los cuales, generan cambios de comportamiento del sujeto producto de estar conectado y expuesto al bombardeo de información durante largas jornadas diarias. Es evidente que, distractores como los videos juegos demandan al sujeto social un elevado consumo de energía, afectando su rendimiento en la vida diaria. Por su parte, este enorme desgaste de energía es una de las principales causantes de la apatía presentada por los estudiantes en el aula de clase (Fuentes y Pérez, 2015). Así mismo, el estar conectados e interactuar con otros actores es una ventana abierta y expuesta a distractores del aprendizaje y de los peligros que existen en la web, (Moral y Suárez, 2016).

Por su parte, (Jasso et al, 2017) expresan la existencia de sitios web implicadas directamente en el desarrollo de conductas adictivas (video juegos), las cuales, se presenta con más frecuencia en individuos con ciertas características de aislamiento social, creándose a partir de ellas el concepto de conductas repetitivas, perdida de autocontrol al uso excesivo de Internet (Lu y Yeo, 2015). Sin embargo, el concepto de adicción solo logró consenso entre expertos hasta la publicación del DMS5³ (2013).

Según el Neurosiquiatra Alfredo Cía (2013) Presidente, Asociación Psiquiátrica de América Latina, manifiesta que, el elemento esencial de todos los trastornos adictivos es la pérdida de control sobre sí mismo, es decir, el descontrol de la persona afectada sobre determinada conducta; cuando la adicción conductual avanza, los comportamientos se vuelven automáticos, en medidaquesonactivados por emociones e impulsos del sujeto con escaso control cognitivo y carencias de autocrítica sobre su comportamiento. No obstante, se teniendo en cuenta que la conducta del sujeto es voluntaria y totalmente consiente, mientras que, el comportamiento puede ser consiente o inconsciente.

Al respecto conviene decir que, al perder el control de la conducta son detonantes de estados de ansiedad, depresión, apatía, pérdida de realidad. Teniendo en cuenta que, terminan generando conductas adictivas, causantes de problemas psicológicos y emocionales del sujeto. Según (BuizaAguado et al., 2017) manifiesta que, el uso de video juegos tiene implicaciones neurobiológicas, psicosociales altamente nocivos para el sujeto social. Mientras que, (Tore Gulden et al., 2017) Sostiene que, la función psicosocial desarrolla un patrón adictivo; presentando más afectaciones al sexo masculino con efectos en factores socio-familiares.

Por otra parte, se respondió la pregunta de esta investigación a través de una búsqueda de literatura científica mediante una revisión bibliométrica

DMS5: Manual Diagnóstico y Estadístico de los Trastornos Mentales de la Asociación Americana de Psiquiatría 
integrativa de las bases de datos Web of Science, Scopus, Dialnet, Redalyc, Scielo y la biblioteca de Multiversidad Mundo Real Edgar Morin. Así mismo, este estudio bibliométrico se fundamentó científicamente en la Ley de crecimiento exponencial de Derek de Solla Price (1963). En su obra, comprueba que la ley del crecimiento de la información científica se produce en forma exponencial y esta crece a un ritmo tan rápido que se duplica entre un rango de 10 a 15 años. No obstante, considera que cada disciplina tiene sus propias etapas de evolución de información científica.

De la misma manera, Price (1963) demuestra con los postulados de la primera ley considera que, la ciencia crece a interés compuesto, multiplicándose por una cantidad determinada en periodos iguales de tiempo y se multiplica a sí misma por 2. Segundo, la tasa de crecimiento es proporcional al tamaño de la población o magnitud total adquirida. De manera que, los enunciados verbales se corresponden con la siguiente expresión:

\section{Matemática se expresa:}

$\mathrm{N}=$ Magnitud medida relacionada con el tamaño de la Ciencia

No = Magnitud medida en el tiempo $t=0$

b. = Constante que relaciona la velocidad de crecimiento con el tamaño ya adquirido de la Ciencia.

\section{Complejidad humana y aprendizaje multidimensional}

La estructura cognitiva del homo sapiens actual es una construcción circular producto del comportamiento simbólico y el lenguaje. Mientras que, el comportamiento simbólico expresa una representación sensorial perceptible de una realidad socio-cultural Rivera (2003). El lenguaje es una manifestación comunicativa del pensamiento como resultado de sentimientos y emociones del sujeto, el cual le permite reflexionar, conceptualizar, describir ideas, (Vygotsky, 1973; Bruner, 1984). Sin embargo, el lenguaje también ha servido para manipular al ser humano a través de su historia llevándolo a ser un esclavo de la realidad que construye a través de sus propias teorías, creencias y mitos, Noah Harari (2014). El autor considera que esta manifestaciones, no está lejos de lo que sucede con la tecnología digital y especialmente con la incidencia del internet y la transformación de la sociedad y la cultura de las nuevas generaciones.

Por lo tanto, el aprendizaje se ha plasmado en conocimientos almacenados en la memoria en la cultura del homo sapiens durante miles de años de existencia. Es decir transforma su estructura cognitiva adaptándose para la supervivencia a través del tiempo, Parejo (2018). En otras palabras, el sujeto social por su naturaleza es un sistema orgánico complejo e irrepetible, pues no hay dos sujetos con la misma información en su estructura cognitiva. Ciertamente, podrían convivir bajo el mismo contexto natural, no obstante, cada individuo a través de los sentidos y mediante estímulos externos de manera subjetiva, construye su propia realidad. Jean Piaget (1977) la explica a través de su epistemología genética, manifestando que el conocimiento procede de la acción ejercida por parte del sujeto sobre los objetos y como resultado de estas interacciones el sujeto construye y reconstruye su conocimiento. 


\section{Sistema auto-eco- organizador y complejidad}

Es esencial comprender que, el ser humano es un sistema complejo que se alimenta de información, materia y energía de su entorno autoeco-organizador. Este concepto es planteado por Morin (1977), manifiesta que todo ser viviente tiene necesidad de automantenerse, renovando y alimentándose de energía fresca de su entorno natural. De manera que, las estructuras de los elementos que conforman un sistema vivo se alimenta de influencia de información recíproca en donde esta puede generar crecimiento, permaneciendo naturalmente en estado de equilibrio o puede moverse hacia un estado de desequilibrio. Además plantea que, en los seres humanos existe un principio de autonomía, de dependencia tanto material como espiritual, no solamente de alimentos materiales, sino también de alimentos culturales, del lenguaje, del saber y de las cosas técnicas y sociales.

Por su parte, Prigogine (1977) explica que los seres vivos no son seres aislados que se relacionan, pues todos los seres vivientes pertenecen a una misma realidad con funciones diferentes. Sostiene que, sujeto y objeto constituyen un sistema único con el cual todas las partes forman un todo orgánico, en donde toda fluctuación tiene entonces consecuencias que se propagan a través de todo el sistema. Esto se debe, a cada individuo necesariamente está en interacción con el contexto que le circunda, lo de adentro conecta con lo de afuera, a su vez, lo de afuera conecta con lo de adentro. Denomina a todos los organismos vivos como estructuras disipativas, la cual constituyen en composiciones coherentes autoorganizadas percibidos como sistemas complejos alejados del equilibrio, plantea que, estas estructuras ordenados demuestra que es posible crear orden del desorden y pequeños cambios pueden conducir a resultados totalmente impredecibles.

\section{Complejidad y sistemas orgánicos complejos}

$\begin{array}{ccc}\text { Ludwig Bertalanffy (1976), } & \text { de la }\end{array}$ complejidad a través de la creación de la teoría de sistemas. Además, la apuesta por llevarlo a la práctica y estructurar el conocimiento y la comprensión de los sistemas, interviene en el florecimiento del pensamiento sistémico. En efecto, esta es una manera de entender el mundo no solamente desde las variables o propiedades del objeto o del sujeto, sino, una oportunidad de ver el mundo como un sistema vivo. En donde, todo tiene que ver con todo, como las acciones que realiza el sujeto afecta las relaciones del entorno natural, social y cultural.

En este sentido, Peter Senge (1990) manifiesta que, la conducta de todos los sistemas vivos sigue ciertos principios comunes o patrones de comportamiento cuya naturaleza se han venido descubriendo y analizando desde las ciencias de la complejidad. Mientras que, Jay Forrester (1992) planteo la necesidad de crear herramientas para la modelación y simulación, que permitan predecir el comportamiento de los sistemas: sociales, biológicos, empresariales, económicos, tecnológicos, entre otros. Como resultado, Forrester (1992) y su grupo de investigación del MIT (Massachusetts Institute of Technology) crea la dinámica de sistemas, la cual 
Tecnología digital y afectaciones a la cultura de aprendizaje del sujeto social Rodriguez-Cadena Rodolfo

representa una herramienta basada en diagramas Forrester que permiten modelar y simular sistemas complejos.

En este sentido, gráficamente se representa la afectación de la tecnología digital al entorno del sujeto social en un sistema dinámico complejo (diagrama 1). Es decir, en el modelo se puede ver reflejado las variables exógenas que inciden en el aprendizaje del sujeto social. Estas variables exógenas están representadas por: la cultura, la sociedad, la familia, la escuela y por último la tecnología digital, que afecta el sistema el entorno auto-eco-organizador del sujeto social. Dado que, el individuo representa un organismo complejo que forma parte de un sistema que construye su estructura cognitiva a través de la incidencia de variables exógenas de su entorno tanto consiento como inconsciente, evidenciando la manera como la tecnología digital irrumpe el entorno auto-eco-organizador del sujeto social.

\section{Diagrama 1 \\ Incidencia de la tecnología en el sistema auto-eco-organizador}

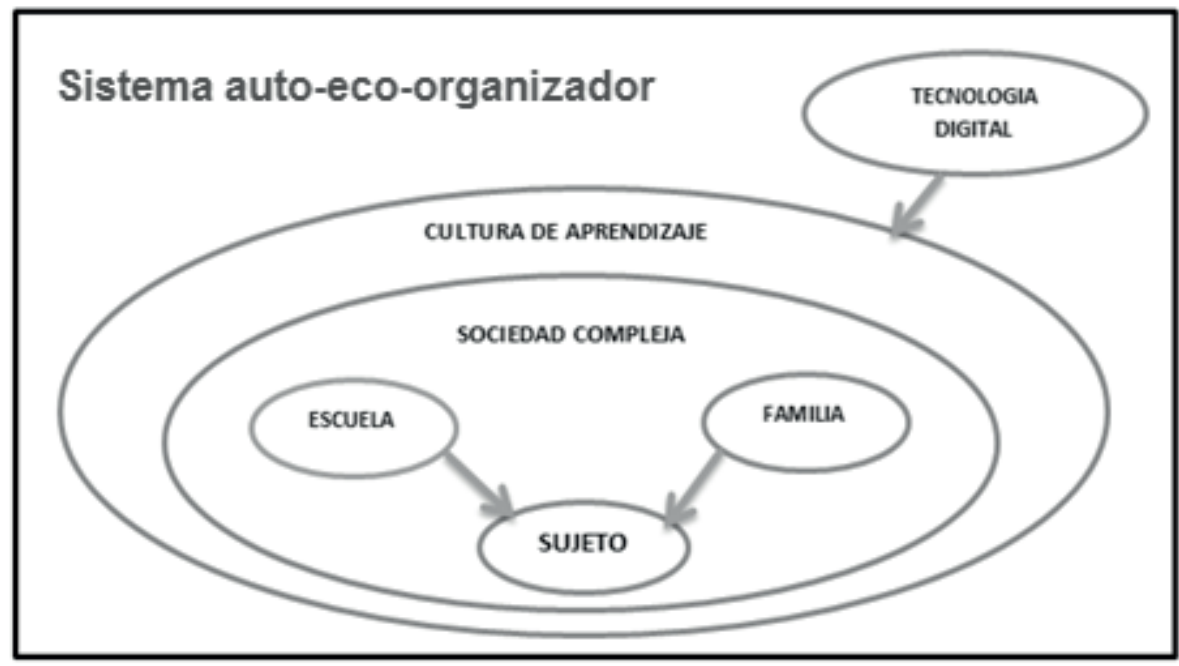

Fuente: Elaboración propia tesis de grado basado en sistema auto-eco-organizador

\section{Bioética, tecnociencia y responsabilidad social}

La humanidad necesita recordar las palabras planteadas por Van Rensselaer Potter (1970), padre de la bioética, quien manifestó su preocupación por los daños ocasionados contra todas las forma de vida biológica por los avances tecnológicos. Manifestaba que, la humanidad necesitaba crear de forma urgente una nueva sabiduría que le proporcionara "el conocimiento de cómo usar el conocimiento, para la 
supervivencia del hombre". Argumentaba que, la bioética es la ética basada en el conocimiento biológico dirigido a la supervivencia de la vida en todo el planeta. Tenía la ilusión que existía la posibilidad de hacer mejor las cosas. Pues creía que era necesaria la creación de una ciencia de la supervivencia basada en el conocimiento biológico que le permitiera alcanzar una mejor visión de la realidad (Pérez-Tamayo, 2015). Sin lugar a dudas, Potter es un visionario de la complejidad para la época al considerar que la nueva ética se deriva de alcanzar la sabiduría para modificar nuestro comportamiento y adaptarnos mejor a nuestro entorno natural y poder sobrevivir.

Otro aspecto esencial es el concepto de responsabilidad social de las empresas dedicadas al diseño y desarrollo de tecnología, las cuales, está fuertemente relacionado con la ética y la moral (Uribe, 2017). En vista que, las decisiones empresariales que toman tanto individuo como organización, afectan tanto al sujeto, como la sociedad tiene en cuenta que existen áreas críticas en las empresas cuyas acciones ocasionan consecuencias imprevisibles para la humanidad. Mientras que, para (Castrillón y Córdoba, 2016:498), la responsabilidad social organizacional plantea reconocer como un resultado ineludible de los fundamentos mismos de la conducta humana, en donde se desarrolla una conciencia de conjunto, manifiestan que, existen tres grandes marcos generales de referencia basados en deberes (el marco de ontológico), el de metas y objetivos (la visión teleológica), y finalmente el de perspectivas axiológicas propuestas.

Las redes sociales son sistemas complejos en la medida que permiten el intercambio de información y energía.
Aunque existen diferencias de tiempo de vida de los sistemas de las organizaciones humanas. Debido a que unas han podido perdurar en el tiempo mucho más que otras. Este fenómeno se da por el principio de entropía ya que la energía de un sistema se va disminuyendo a lo largo del tiempo hasta dejar de existir Prigogine (1974). Además, las redes sociales son sistemas de comunicación que al interactuar seres vivos implican un proceso de autoorganización que involucra una evolución a un sistema mucho más organizados en donde emergen estructuras o patrones de comportamiento más complejos.

\section{Análisis resultados bibliométrico de la investigación}

Dentro de la búsqueda bibliométrica se destaca las afectaciones que ha generado el mal uso de la tecnología digital a la cultura de aprendizaje de las nuevas generaciones. Se destaca un número muy significativo de literatura científica que aborda temáticas relacionadas con las afectaciones de la tecnología digital al ser humano y las afectaciones al sujeto con los siguientes hallazgos:

Adicción a internet: caracteriza por la pérdida de control y un fuerte impulso y necesidad subjetiva de usar internet o una aplicación específica, a pesar de las consecuencias negativas del uso. La adicción al internet presenta algunas características similares a otras adicciones conductuales como las adicciones a las sustancias alucinógenas. Por otra parte, es significativo señalar la inclusión en el DSM-5 (APA, 2013) el cual reconoce como desordenes de juegos a través 
Tecnología digital y afectaciones a la cultura de aprendizaje del sujeto social Rodriguez-Cadena Rodolfo

del internet ocasionando los siguientes trastornos:

Trastorno de ansiedad: Depresión Cuestionario Goldberg

Trastorno de depresión: Depresión Cuestionario Goldberg

Perdida de realidad y apatía a aula de clase: causada por el consumo de energía por la exposición a largas jornadas frente a dispositivos electrónicos.

Sin embargo, lo más significativo de los hallazgos encontrados son los patrones de comportamiento del sistema auto-eco-organizador conformado por los investigadores que realizaron estas investigaciones durante los últimos 15 años, los resultados demuestran el comportamiento estadístico de los periodos comprendidos entre el año 2004 y 2018 periodo en cual ha generado una producción científica de 5856 publicaciones agrupados por variables comunes como las afectaciones por el mal uso de la tecnología. Los resultados de las publicaciones se distribuyeron de la siguiente manera: 942 capítulos de libro de investigación, 795 artículos de revisión y 4241 artículos de investigación, ver diagrama 2.

\section{Diagrama 2 \\ Representación gráfica de los resultados}

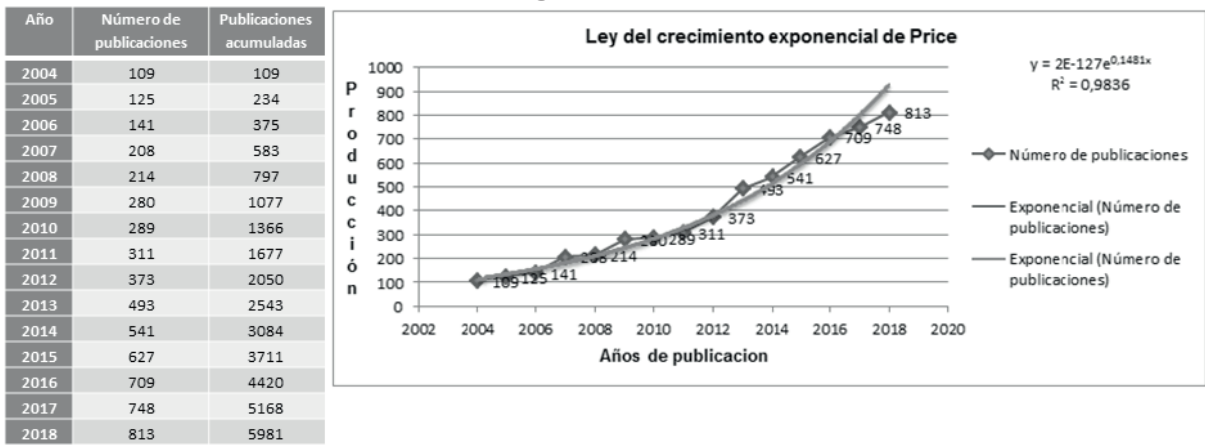

Fuente: Elaboración propia ley crecimiento exponencial de Derek De Solla Price (1956)

La ecuación exponencial

$$
\begin{aligned}
& Y=2 E-127 e^{0.1481 x} \\
& R^{2}=0.9836
\end{aligned}
$$

Se debe tener presente que, el valor es de 0.9836 , es decir que este modelo exponencial representa los ingresos en relación con los años en un $98.36 \%$ el valor tiene una alta correlación entre el número de publicaciones por año recordando que entre más cercano a 1 el valor de R2 los datos tendrán un ajuste más apropiado.

\section{Análisis resultados desde la postura complejidad}

De acuerdo con la postura del pensamiento complejo Edgar Morin 
(2008), el pensamiento del hombre actual es el resultado de una mirada sesgada de la ciencia clásica, desplegada entre los siglos $\mathrm{XVII}$ y finales del siglo $\mathrm{XX}$. Por lo tanto, las teorías de aprendizaje emprendidas por la investigación científica durante los últimos cien años, exponen una mirada disciplinar desde diferentes posturas conductistas, cognitivistas, constructivistas, neuroaprendizaje o desde el aprendizaje activo. Sin embargo, este tipo de propuestas teóricas del aprendizaje son miradas disciplinares que mutilan las dimensiones y complementariedades del ser humano. Esto evidencia que, la mirada disciplinar de la tecnociencia exponen una realidad presente el principio filosófico de la biótica de Potter (1970). Quien argumentaba la necesidad de "tener conocimiento de cómo usar el conocimiento, para la supervivencia del hombre".

Es necesario comprender que, afrontar el aprendizaje del homo sapiens no es una tarea disciplinar como lo ha venido haciendo las ramas de la ciencias de la educación en el siglo XX. Por lo tanto, emerge una necesidad conocer y comprender las complejidades y dimensiones del homo sapiens. Es decir, abordar el aprendizaje humano implica hacerlo desde una mirada integradora de saberes interdisciplinarios; en el cual se pueda emprender el estudio del aprendizaje humano desde biológico, psicológico, sociológico y lo antropológico, con el propósito de crear una verdadera ciencia integradora de saberes y el desarrollo de una nueva pedagogía basada en la síntesis para el desarrollo de la metacognición más allá de los conceptos.

\section{Conclusiones}

El estudio de esta problemática que busca identificar las afectaciones a la cultura de aprendizaje de las nuevas generaciones, se puede percibir como que emerge la necesidad de abordar los problemas desde la visión de los sistemas complejos. Teniendo en cuenta que, es una mirada holística en donde se relaciona e interrelacionada el todo con sus partes, pero que también, involucra las variables exógenas que afectan al sujeto social. Por lo tanto, desde la bioética involucra la responsabilidad social que deben tener las organizaciones cuando desarrollan tecnología. En donde, es fundamental comprender que cuando se diseña y desarrolla tecnología, se debe tener presente las afectaciones que esta genera al sujeto social.

Desde su origen evolutivo el homo sapiens logro sobrevivir a los peligros de época gracias a su capacidad de adaptación al contexto ecológico. Este hecho es muy significativo, porque fue a través del lenguaje y el pensamiento simbólico, que el hombre alcanza una ventaja estratégica sobre las demás especies del planeta, al emerger la capacidad de relacionar e interrelacionar con los sujetos de su misma especie. Sin embargo, la tecnología digital y el internet están consiguiendo un efecto contrario al incidir en el aislamiento del sujeto social, desequilibrando su nicho ecológico e incidiendo en el estado emocional y cambio de comportamiento del sujeto social.

Para finalizar, desde la búsqueda bibliométrica de la ley del crecimiento exponencial propuesto Derek de Solla Price (1956) queda demostrado que los problemas que generara la incidencia de la tecnología digital sobre los seres humanos en los últimos 15 años la afectaciones al sujeto social y la cultura de aprendizaje se convertirán en una disciplina de 
Tecnología digital y afectaciones a la cultura de aprendizaje del sujeto social

Rodriguez-Cadena Rodolfo

investigación de salud pública para la ciencia desde las multidisciplinariedad, interdisciplinariedad

transdisciplinariedad por explorar en los próximos años.

\section{Referencias bibliográficas}

Arab, Elías y Díaz, Alejandra (2015), Impacto de las redes sociales e internet en la adolescencia: aspectos positivos y negativos." Revista Médica Clínica Las Condes 26 (1): 7-13. doi:10.1016/j.rmclc.2014.12.001.

Asociación Americana de Psiquiatría (2013), Diagnóstico y estadístico de los trastornos mentales (American Psychiatric Association, APA), 5th Edición (DSM-5). Washing- ton, DC.

Bell, Daniel (1976), The coming of Post-Industrial Society A venture in social fore casting, Harmondsworth, Peregrine.

Buiza-Aguado, Carlos; García-Calero Alfonso; Alonso-Cánovas Araceli; Ortiz-Soto, Paloma; Guerrero-Díaz Miguel; González-Molinier, Manuel y Hernández-Medrano Ignacio (2017), Los videojuegos: una afición con implicaciones neuropsiquiátricas. Psicología Educativa 23 (2): 129-36. https://doi.org/10.1016/j. pse.2017.05.001.

Bruner, Jerome (1984), Acción pensamiento y lenguaje, Madrid: Alianza Editorial.

Carbonell, Xavier (2014), La adicción a los videojuegos en el DSM-5, Vol. 26, Núm (2), 91-95, Universidad Ramón Llull, FPCEE Blanquerna. Barcelona, España.

Cuzin, Laoin (2007), Collective minds, Nature. Nature Publishing Group, 445, p. 715. Available at: https://doi. org/10.1038/445715a.
De Solla Price, Derek (1963), Little Science, big science. New York: Columbia University Press.

Forrester, Jay (1992), La dinámica de sistemas y el aprendizaje del alumno en la educación escolar: proyecto educativo dinámica de sistemas, grupo de dinámica de sistemas, Escuela de Administración Massachusetts, Institute of Technology.

Fuentes Fuentes, Luz Stella y Pérez Castro, Libia María (2015), Los videojuegos y sus efectos en escolares de Sincelejo, Sucre (Colombia). Opción [en linea]., 31(6), 318-328[fecha de Consulta 1 de Enero de 2019]. ISSN: 1012-1587. Disponible en: https://www.redalyc. org/articulo.oa?id=31045571020

Jasso, Jose; López, Fuensanta y Díaz, Rolando (2017), Conducta adictiva a las redes sociales y su relación con el uso problemático del móvil, Acta de investigación psicológica, https://doi.org/10.1016/j.aipprr.2017.11.001.

Lu, Xi y Kee Jiar, Yeo (2015), Propiedades psicométricas del test de adicción a internet en una muestra de estudiantes universitarios malayos, Psicología Educativa 21 (1): 17-25, https://doi.org/10.1016/j. pse.2015.03.001.

Maturana, Humberto y Varela, Francisco (2003), De máquinas y seres vivos: autopoiesis, la organización de lo vivo, Chile, Editorial Universitaria s.a.

Maldonado, Carlos Eduardo (2009), "Complejidad de los Sistemas Sociales: Un reto para las ciencias sociales", Revista ciencias sociales: Cinta Moebio, No 36: 146-157 www.moebio.uchile.cl/36/maldonado.html

McLuhan, Marshall (1995), La aldea global, Barcelona: Editorial Gedisa. 
Morin, Edgar (1977), El método I: La naturaleza de la naturaleza, Madrid, Cátedra, 1981 Hermosillo, Sonora, Multiversidad Mundo Real, consultado el día 8 de abril del 2019, Bajado de: www.http://multiversidadreal.edu. $\mathrm{mx}$

Moral, Maria y Suárez, Cecilia (2016), Factores de riesgo en el uso problemático de Internet y del teléfono móvil en adolescentes españoles. Revista Iberoamericana de Psicología y Salud , 7 (2), 69-78. https://doi. org/10.1016/j.rips.2016.03.001

Muñoz-Dueñas, Carmen; Burgos-Muñoz, Sara; Novoa-Sandoval, Paola y Toro-Huamanchumo, Carlos (2017), Adicción a Internet: ¿cuál es la realidad en estudiantes de medicina de Latinoamérica?, Educación médica: Fundación educación médica. https://doi.org/10.1016/j.edumed.2017.01.005

Parejo, Alexander (2018), Medición del desarrollo de competencias interpretativas, argumentativas y propositivas mediadas con TIC. Opción, Año 34, Especial No.18 (2018): 1740-1764

Peter, Senge (1995), La quinta disciplina: cómo impulsar el aprendizaje en la organización inteligente, Ediciones Granica S.A. Buenos Aires Argentina.

Pérez-Tamayo, Ruy (2015), La bioética en México hoy, Alicante: Biblioteca Virtual Miguel de Cervantes, 2015. Consultado en: http://www. cervantesvirtual.com/nd/ark:/59851/ bmcg7591

Pedrero-Pérez, Eduardo; Ruiz-Sánchez, Jose; Rojo-Mota, Gloria; Llanero-Luque, Marcos; Pedrero-Aguilar, Jara; Morales-Alonso, Sara y Puerta-García, Carmen (2018), Tecnologías de la Información y la Comunicación (TIC): Uso problemático de in- ternet, videojuegos, teléfonos móviles, mensajería instantánea y redes sociales mediante Vol. 30, 19-32, doi:10.20882/adicciones.806

Piaget, Jean (1977), Piaget en Piaget: la epistemología de Jean Piaget, Editor [New Haven, Connecticut]: Yale University Media Design Studio [compañía de producción]Dirigido por Philip. Garvin. Producido por Philip Garvin. Consultado: https://archive.org/details/piagetonpiagettheepistemologyofjeanpiaget

Potter, Van Rensselaer (1970), Bioética: La ciencia de la supervivencia, New Jersey: editorial Prentice Hall.

Prigogine, Ilya; Nicolis, G. (1977), Autoorganización en sistemas no equilibrados.

Piaget, Jean (1991), seis estudios de psicología, Barcelona: Labor S.A.

Rodríguez, Leonardo y Aguirre, Leónidas (2011), "Teorías de la complejidad y ciencias sociales. Nuevas estrategias epistemológicas y metodológicas", Nómadas No 30 (2): 14766. doi:10.5209/rev_NOMA.2011. v30.n2.36562.

Rivas-Peña, Pedro y Bello-Larreal, Robert (2017), Liderazgo Bioético: Una Cosmovisión Sistémica en las Organizaciones Complejas. Revista Venezolana de Gerencia, Año. 22, No. 77, 2017 pp 24-35

Solla Price, Derek (1963), Little Science Big Science, New York: Columbia University Press.

Tello Castrillón, Carlos y Rodriguez, María del Pilar (2016), "Fundamentos Éticos Disciplinares de La Responsabilidad Social Organizacional." Revista Venezolana de Gerencia RVG 75 (508): 491-508.

Tore Gulden, Maríal; Cecilia Suárez; Casas, Rosario Ruiz-Olivares, Ro- 
Tecnología digital y afectaciones a la cultura de aprendizaje del sujeto social

Rodriguez-Cadena Rodolfo

sario Ortega-Ruiz, Xi Lu, et al. 2017. Factores de riesgo en el uso problemático de Internet y del teléfono móvil en adolescentes españoles. Psicología Educativa 7 (2): 79-85. https://doi.org/10.1016/j. aprim.2013.06.001.

Yazdani, Naveed \& Murad, Hasan (2014), Toward an ethical theory of organizing. Journal of Business Ethics, 127(2), 399-417.

Vygotski, Lev (1973), Pensamiento y Lenguaje: Teoría del desarrollo cultural de las funciones psíquicas, Buenos Aires, Pléyade, Ediciones Fausto, Traducción del original ruso: María M. Rotger, (pp. 9-348), Trabajo original publicado 1934.

Villa Moral, María y Suárez, Cecilia (2016), Factores de riesgo en el uso problemático de Internet y del teléfono móvil en adolescentes españoles, Revista Iberoamericana de Psicología y Salud, Volumen 7, Pages 69-78. https://doi.org/10.1016/j. rips.2016.03.001.(http://www.sciencedirect.com/science/article/pii/ S2171206916300011).
Solana Ruiz, José Luis (2011), El pensamiento complejo de Edgar Morin, Críticas, incomprensiones y revisiones necesarias, Gazeta de Antropología, № 27 /1, 2011, Artículo 09.

Uribe, Adriana; Blanco, Ana; Peralta, Pablo; Zapata, Álvaro; Daza, Alexander; Monterrosa Iván; Cervantes Álvaro; Lombana Jahir; Zapateiro Orlando; Taboada Ramón; Aylin Pertuz; Mulford Marelys; Molinares Carlos; Diana Urrego; Frank Toala; Prieto Francia (2017). La investigación en el programa de Administración de Empresas de la Universidad Simón Bolívar, Barranquilla. En A. Uribe, La investigación en los programas de Administración de Empresas del Caribe Colombiano Barranquilla: Ediciones Universidad Simón Bolívar.

Von Bertalanffy, Ludwig (1976), Teoría general de los sistemas, Fundamentos, desarrollo, aplicaciones, México: Fondo de Cultura Económica.

- Esta obra está bajo una licencia de Creative Commons Reconocimiento-NoComercialCompartirlgual 3.0 Unported. http://creativecommons.org/licenses/by-nc-sa/3.0/deed.es_ES 